\title{
Knockdown of long noncoding RNA HOTAIR inhibits osteoarthritis chondrocyte injury by miR-107/CXCL12 axis
}

\author{
Jipeng Lu, Zhongxiong $\mathrm{Wu}^{*}$ and Ying Xiong
}

\begin{abstract}
Background: Osteoarthritis $(\mathrm{OA})$ is a joint disease characterized via destruction of cartilage. Chondrocyte damage is associated with cartilage destruction during OA. Long noncoding RNAs (IncRNAs) are implicated in the regulation of chondrocyte damage in OA progression. This study aims to investigate the role and underlying mechanism of IncRNA homeobox antisense intergenic RNA (HOTAIR) in OA chondrocyte injury.
\end{abstract}

Methods: Twenty-three OA patients and healthy controls without OA were recruited. Chondrocytes were isolated from OA cartilage tissues. HOTAIR, microRNA-107 (miR-107) and C-X-C motif chemokine ligand 12 (CXCL12) levels were measured by quantitative real-time polymerase chain reaction and western blot. Cell proliferation, apoptosis and extracellular matrix (ECM) degradation were measured using cell counting kit-8, flow cytometry and western blot. The target interaction was explored by bioinformatics, luciferase reporter and RNA immunoprecipitation assays.

Results: HOTAIR expression was enhanced, and miR-107 level was reduced in OA cartilage samples. HOTAIR overexpression inhibited cell proliferation, but induced cell apoptosis and ECM degradation in chondrocytes. HOTAIR knockdown caused an opposite effect. MiR-107 was sponged and inhibited via HOTAIR, and knockdown of miR-107 mitigated the effect of HOTAIR silence on chondrocyte injury. CXCL12 was targeted by miR-107. CXCL12 overexpression attenuated the roles of miR-107 overexpression or HOTAIR knockdown in the proliferation, apoptosis and ECM degradation. CXCL12 expression was decreased by HOTAIR silence, and restored by knockdown of miR-107.

Conclusion: HOTAIR knockdown promoted chondrocyte proliferation, but inhibited cell apoptosis and ECM degradation in OA chondrocytes by regulating the miR-107/CXCL12 axis.

Keywords: Osteoarthritis, Chondrocyte, HOTAIR, miR-107, CXCL12

\section{Introduction}

Osteoarthritis (OA) is one of the most common joint diseases, which can involve all joint tissues, such as the subpatellar fat pad, synovial membrane, subchondral bone, meniscus, ligaments and cartilage $[1,2]$. Chondrocytes are integral to the anabolic-catabolic balance in the cartilage, and their abnormalities are responsible for

\footnotetext{
*Correspondence: spring_beginning@163.com

* Correspondence: spring_beginning@163.com
Department of Orthopedics, Yan'an Hospital Affiliated to Kunming Medical University, No. 245 Renmin East Road, Panlong District, Kunming 650051, Yunnan, China
}

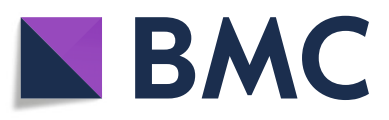

(0) The Author(s). 2021 Open Access This article is licensed under a Creative Commons Attribution 4.0 International License, which permits use, sharing, adaptation, distribution and reproduction in any medium or format, as long as you give appropriate credit to the original author(s) and the source, provide a link to the Creative Commons licence, and indicate if changes were made. The images or other third party material in this article are included in the article's Creative Commons licence, unless indicated otherwise in a credit line to the material. If material is not included in the article's Creative Commons licence and your intended use is not permitted by statutory regulation or exceeds the permitted use, you will need to obtain permission directly from the copyright holder. To view a copy of this licence, visit http://creativecommons.org/licenses/by/4.0/. The Creative Commons Public Domain Dedication waiver (http://creativecommons.org/publicdomain/zero/1.0/) applies to the data made available in this article, unless otherwise stated in a credit line to the data.

the destruction of cartilage during OA [3]. Chondrocyte is the only cell type of cartilage, and the apoptosis and extracellular matrix $(\mathrm{ECM})$ degradation of chondrocytes are related to OA progression $[4,5]$. Therefore, exploring the mechanism of chondrocyte injury might indicate new insight into the pathology of OA, and provide novel target for OA treatment.

Noncoding RNAs are involved in cartilage development and chondrocyte injury [6, 7]. Long noncoding RNAs (lncRNAs; with > 200 nucleotides in length) are a type of noncoding RNA that can be involved in cartilage 
development and degeneration, and have important values in the treatment of bone-related diseases, including OA $[8,9]$. Moreover, IncRNAs play important roles in OA progression by regulating chondrocyte processes, like proliferation, apoptosis, inflammation and ECM [10]. LncRNA homeobox antisense intergenic RNA (HOTAIR) is implicated in multiple cell biological processes, including proliferation, migration, invasion and apoptosis by acting as a competitive endogenous RNA (ceRNA) through interacting with microRNAs (miRNAs) $[11,12]$. A previous report revealed that HOTAIR could increase chondrocyte apoptosis which was regulated via lncRNA P50-associated cyclooxygenase-2 extragenic RNA (PACER) [13]. Furthermore, HOTAIR might promote OA progression by regulating ECM degradation and chondrocyte apoptosis through the miR-17-5p/fucosyltransferase 2 (FUT2)/ $\beta$-catenin axis [14]. Nevertheless, the mechanisms are complex, and more regulatory networks mediated by HOTAIR in chondrocyte damage during OA progression are needed to be explored.

MiRNAs are a class of noncoding RNAs with $\sim 22 \mathrm{nu}$ cleotides interacting with cognate mRNAs, which play key roles in OA progression [15]. A former study suggested that miR-107 could mitigate chondrocyte apoptosis and ECM degradation via regulating phosphatase and tensin homolog deleted on chromosome ten (PTEN) [16]. Zhao et al. reported that miR-107 could inhibit apoptosis and promote autophagy of chondrocytes during $\mathrm{OA}$ progression by regulating tumor necrosis factor receptor associated factor three (TRAF3) [17]. However, whether miR-107 is responsible for HOTAIR to regulate chondrocyte damage during OA remains unclear. Moreover, many differentially expressed genes are involved in the development of chondrogenesis during OA [18], in which C-X-C motif chemokine ligand 12 (CXCL12) has been reported to participate in the development of some inflammatory diseases [19]. Bioinformatics assay showed miR-107 could bind with HOTAIR and CXCL12. Thus, we hypothesized HOTAIR might regulate CXCL12 by competitively binding with miR-107. However, the HOTAIR/miR-107/CXCL12 axis has not been reported in OA progression.

In an attempt to elucidate the function and regulatory mechanism of HOTAIR in OA progression, this research focused on the effect on cell proliferation, apoptosis and ECM degradation in OA chondrocytes, and investigated the regulatory network of HOTAIR/miR-107/CXCL12.

\section{Materials and methods}

\section{Cartilage tissues collection}

Twenty-three OA knee cartilage samples were harvested from OA patients who underwent total knee arthroplasty. The normal knee cartilage samples were obtained from 23 patients with surgical repair of fractures, who did not have OA. The characteristics of all subjects are shown in Table 1. All patients recruited in this research have signed the informed consents. The study was approved by the ethics committee of Yan'an Hospital Affiliated to Kunming Medical University.

\section{Cell culture and transfection}

Chondrocytes were isolated from knee cartilage tissues of $3 \mathrm{OA}$ patients for 3 independent experiments using collagenase digestion method as previously reported $[20$, 21]. In brief, the inflammatory cartilage tissues were minced, digested with trypsin (Sigma-Aldrich, St. Louis, $\mathrm{MO}$, USA) at $37^{\circ} \mathrm{C}$ for $30 \mathrm{~min}$, and then incubated with $0.2 \%$ collagenase II (Sigma-Aldrich) at $37{ }^{\circ} \mathrm{C}$ for $6 \mathrm{~h}$. After centrifugation at $1500 \mathrm{~g}$ for $10 \mathrm{~min}$, cells were maintained in DMEM (Thermo Fisher Scientific, Waltham, MA, USA) containing $10 \%$ fetal bovine serum at $37{ }^{\circ} \mathrm{C}$ under $5 \% \mathrm{CO}_{2}$. The third passages of cells were used in this research.

Small interfering RNA (siRNA) against HOTAIR (siHOTAIR), siRNA negative control (si-NC), pcDNA-based HOTAIR overexpression vector (HOTAIR), pcDNAbased CXCL12 overexpression vector (CXCL12), pcDNA empty vector, miR-107 mimic (miR-107), miRNA negative control (miR-NC), miR-107 inhibitor (in-miR-107) and inhibitor negative control (in-miR-NC) were synthesized from Genomeditech (Shanghai, China). The chondrocytes were transfected with $1 \mu \mathrm{g}$ vectors or $40 \mathrm{nM}$ oligos using Lipofectamine $^{\mathrm{TM}} 2000$ Transfection Reagent (Thermo Fisher Scientific) for $6 \mathrm{~h}$. After $24 \mathrm{~h}$, chondrocytes were collected for subsequent experiments.

Quantitative real-time polymerase chain reaction (qRT-PCR) Total RNA was isolated using Trizol (Thermo Fisher Scientific). The reverse transcription was performed with 1 mg RNA using All-in-One ${ }^{\mathrm{TM}}$ first strand cDNA

Table 1 Baseline characteristics of the study subjects

\begin{tabular}{lll}
\hline Characteristics & Normal $(\mathbf{n}=\mathbf{2 3})$ & OA $(\mathbf{n}=\mathbf{2 3})$ \\
\hline Age & 12 & 13 \\
$>$ 60 years & 11 & 10 \\
$\quad$ 60 years & 10 & \\
Gender & 13 & 9 \\
Male & & 14 \\
Female & - & \\
OA grading & - & 3 \\
Grade I & - & 11 \\
Grade II & - & 8 \\
Grade III & $24.6 \pm 3.1$ & 1 \\
Grade IV & & $27.4 \pm 2.7$ \\
BMI & &
\end{tabular}

BMI Body mass index 
synthesis kit (FulenGen, Guangzhou, China). The cDNA product was diluted by 1:10, and used for qRT-PCR with specific primers and SYBR Green (Vazyme, Nanjing, China). The qRT-PCR was conducted on CFX96 ${ }^{\text {tm }}$ Realtime PCR Detection System (Bio-Rad, Hercules, CA, USA). The primers used in this research were generated by Sangon Biotech (Shanghai, China) as follows: HOTAIR (forward, 5'-GTTTGGGATCTGTTCCAG CCT-3'; reverse, 5'-CGTCTGTAACTCTGGGCTCC-3'); CXCL12 (forward, 5'-CTACAGATGCCCATGCCGAT3'; reverse, 5'-GTGGGTCTAGCGGAAAGTCC-3'); $\beta$ actin (forward, 5'-CTTCGCGGGCGACGAT-3'; and reverse, 5'-CCACATAGGAATCCTTCTGACC-3'). The primers of miR-107 and U6 were purchased from FulenGen (HmiRQP0030 and HmiRQP9001). Using $\beta$-actin or U6 as reference control, the relative RNA expression was analyzed via the $2^{-\Delta \Delta \mathrm{Ct}}$ method [22].

\section{Cell proliferation assay}

The cell counting kit-8 (CCK-8) kit (Solarbio, Beijing, China) was used for measurement of chondrocyte proliferation. Chondrocytes $\left(3 \times 10^{3}\right.$ cells $)$ were inoculated into 96-well plates in triplicate. Cells were cultured for $0,24,48$ and $72 \mathrm{~h}$, and then interacted with $10 \mu \mathrm{L} \mathrm{CCK}$ 8 solution for another $3 \mathrm{~h}$. Subsequently, the optical density (OD) value at $450 \mathrm{~nm}$ of each well was measured using a microplate reader (Potenov, Beijing, China).

\section{Cell apoptosis assay}

The detection of cell apoptosis was performed with Annexin V-FITC/PI apoptosis detection kit (Vazyme) through flow cytometry. Chondrocytes $\left(5 \times 10^{4}\right.$ cells $)$ were plated into 6-well plates and grown at $37^{\circ} \mathrm{C}$ for 72 h. Then cells were collected and double stained with 10 $\mu \mathrm{L}$ Annexin V-FITC and PI under condition void of light for $10 \mathrm{~min}$. The stained cells were measured with a NovoCyte 1040 flow cytometer (Agilent Biosciences, Hangzhou, China), and the apoptotic rate was expressed as the percentage of cells in the lower and upper right.

\section{Bioinformatics analysis and luciferase reporter assay}

Bioinformatics analysis using DIANA tools was performed, and showed the binding sites of miR-107 and HOTAR or CXCL12 3'UTR. Based on pmirGLO vectors (Promega, Madison, WI, USA), the sequences of HOTAIR or CXCL12 3' UTR with miR-107 binding sites or mutant sites was used to generate wild-type (WT) or mutant (MUT) luciferase reporter vectors, named as HOTAIR WT, HOTAIR MUT, CXCL12 3'UTR WT or CXCL12 3'UTR MUT, respectively. miR-107 mimic, miR-NC, in-miR-107 or in-miR-NC was co-transfected with the luciferase reporter vectors into chondrocytes using Lipofectamine ${ }^{\mathrm{TM}} 2000$ Transfection Reagent. Cells were collected at $24 \mathrm{~h}$ after the transfection, and the relative luciferase activity was detected with a luciferase reporter assay kit (Promega).

\section{RNA immunoprecipitation (RIP)}

The RIP assay was conducted in chondrocytes, with the Magna RNA immunoprecipitation kit (Millipore, Billerica, MA, USA). Cells $\left(1 \times 10^{7}\right)$ were lysed, and then incubated with RIP immunoprecipitation buffer containing magnetic beads pre-coated with Ago2 antibody for $8 \mathrm{~h}$. IgG was regarded as control. The anti-Ago2 (ab32381) was purchased from Abcam (Cambridge, MA, USA), and anti-IgG (AP112) was obtained from Sigma-Aldrich. The level of HOTAIR enriched in complex was detected by qRT-PCR.

\section{Western blot}

The cartilage tissues or chondrocytes were lysed using RIPA buffer with 1\% PMSF (Beyotime) on ice. The BCA Protein Assay Kit (Beyotime) was applied to quantify protein, and then proteins were denaturalized by boiling water bath. The electrophoresis and membrane transfer were performed using Bio-Rad Bis-Tris Gel system (BioRad) following the manufacturer's instructions. After blocking using 5\% nonfat milk, the Abcam primary antibodies were used, including anti-Aggrecan (ab194594, 1: 1000 dilution), anti-Collagen II (ab185430, 1:2000 dilution), anti-MMP-13 (ab1010, 1:2000 dilution), antiMMP-9 (ab119906, 1:2000 dilution), anti-CXCL12 (ab18919, 1:1000 dilution) or anti- $\beta$-actin (ab8227, 1: 5000 dilution) and corresponding secondary antibody (ab6721, ab6728, 1:10000 dilution). The signals were developed using BeyoECL Plus (Beyotime) and then analyzed via Image Lab software (Bio-Rad).

\section{Statistical analysis}

GraphPad Prism 6 software (GraphPad Inc., La Jolla, CA, USA) was used for statistical analysis. The results of 3 repeats were shown as mean \pm standard deviation (S.D.). The linear correlations among HOTAIR, miR-107 and CXCL12 in OA cartilage were investigated by Spearman's correlation analysis. Comparisons of two groups or multiple groups were performed using Student's $t$ test or one-way ANOVA. $P$ value $<0.05$ was considered statistically significant.

\section{Results}

The expression of HOTAIR is upregulated, and miR-107 level is downregulated in OA cartilage

To explore the role of HOTAIR and miR-107 in OA progression, their expression levels were measured in 23 OA cartilage tissues and normal samples. As shown in Fig. 1A, the expression of HOTAIR was significantly elevated in $\mathrm{OA}$ tissues compared with that in the normal group. Moreover, miR-107 level was obviously lower in 

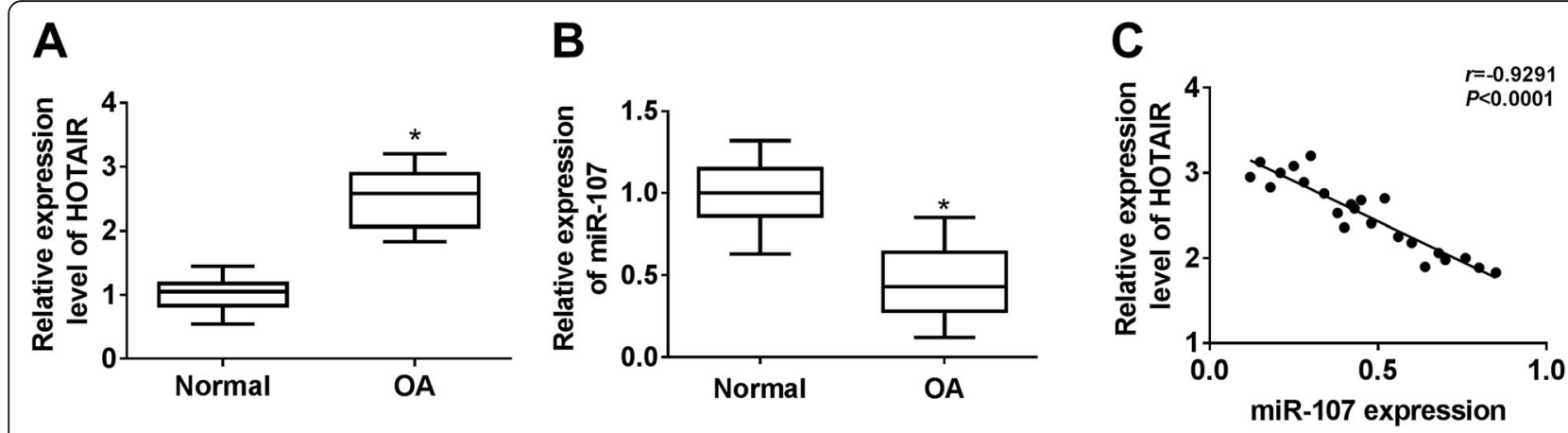

Fig. 1 The expression of HOTAIR and miR-107 in OA. A and B The expression levels of HOTAIR and miR-107 were measured in OA and normal tissues by qRT-PCR. C The correlation of abundance of HOTAIR and miR-107 in OA tissues was investigated by Spearman's correlation analysis. $\mathrm{n}$ $=23 .{ }^{*} P<0.05$

OA cartilage than that in normal samples (Fig. 1B). Meanwhile, the expression of miR-107 in OA cartilage tissues had a negative correlation with HOTAIR level $(\mathrm{r}=-0.9291, P<0.0001)$ (Fig. 1C). These findings suggested that HOTAIR and miR-107 might play important roles in OA progression.

\section{HOTAIR regulates chondrocyte proliferation, apoptosis and ECM degradation}

To investigate the biological role of HOTAIR in vitro, its expression in chondrocytes from OA patients was upregulated by transfection of HOTAIR overexpression vector and downregulated using siRNA, which was validated by qRT-PCR (Fig. 2A). The results of CCK- 8 displayed that after the culture of $72 \mathrm{~h}$, chondrocyte proliferation was significantly decreased by overexpression of HOTAIR, but promoted by HOTAIR knockdown in comparison with their corresponding controls (Fig. 2B). In addition, the data of flow cytometry revealed in Fig. $2 \mathrm{C}$ that addition of HOTAIR induced great increase of apoptotic rate of chondrocytes, whereas silencing HOTAIR led to reduction of cell apoptosis. Moreover, HOTAIR overexpression promoted the protein expression of MMP-13 and MMP-9, but decreased the levels of Aggrecan and Collagen II in chondrocytes (Fig. 2D). However, HOTAIR knockdown led to an opposite effect. These results indicated that HOTAIR knockdown increased cell proliferation, but suppressed chondrocyte apoptosis and ECM degradation.

\section{HOTAIR regulates chondrocyte proliferation, apoptosis and ECM degradation by sponging miR-107}

To explore how HOTAIR affected OA progression, its potential targets were explored by bioinformatics analysis using DIANA tools. We selected 5 downregulated targets (miR-107, miR-197-3p, miR-136-5p, miR-211-5p and $\mathrm{miR}-17-5 \mathrm{p}$ ) in $\mathrm{OA}$ according to previous reports
[17, 23-26]. Furthermore, miR-107 expression was decreased most by HOTAIR overexpression than other 4 miRNAs (Supplementary Figure 1A). Hence, miR-107 was selected for further study. As shown in Fig. 3A, the potential binding sites of HOTAIR and miR-107 were predicted, suggesting that miR-107 might be bound to HOTAIR. To prove this prediction, HOTAIR WT or HOTAIR MUT luciferase reporter vectors were generated, and transfected into chondrocytes. Results showed that overexpression of miR-107 notably reduced the luciferase activity in cells transfected with HOTAIR WT vectors, while its deficiency showed an opposite effect (Fig. 3B, C). However, overexpressing or knocking down miR-107 could not affect the luciferase activity in the HOTAIR MUT group. Moreover, the data of RIP assay revealed that HOTAIR and miR-107 were enriched by Ago2 RIP compared with that in the IgG group (Fig. 3D). Additionally, miR-107 expression level was increased by silencing HOTAIR, and decreased by HOTAIR overexpression in chondrocytes (Fig. 3E). These results suggested HOTAIR as a sponge of miR107 in chondrocytes.

To investigate whether miR-107 was required for HOTAIR-mediated OA progression, chondrocytes were transfected with si-NC, si-HOTAIR, si-HOTAIR + inmiR-NC or in-miR-107, followed by investigation of cell function. As demonstrated in Fig. 4A, miR-107 exhaustion abrogated silencing HOTAIR-mediated promotion of proliferation in chondrocytes. Furthermore, knockdown of miR-107 attenuated downregulation of HOTAIR-mediated apoptosis inhibition in chondrocytes (Fig. 4B). Besides, inhibition of miR-107 weakened knockdown of HOTAIR-mediated upregulation of Aggrecan and Collagen II as well as downregulation of MMP-13 and MMP-9 (Fig. 4C). These findings indicated that HOTAIR mediated chondrocyte injury relying on the regulation of miR-107. 


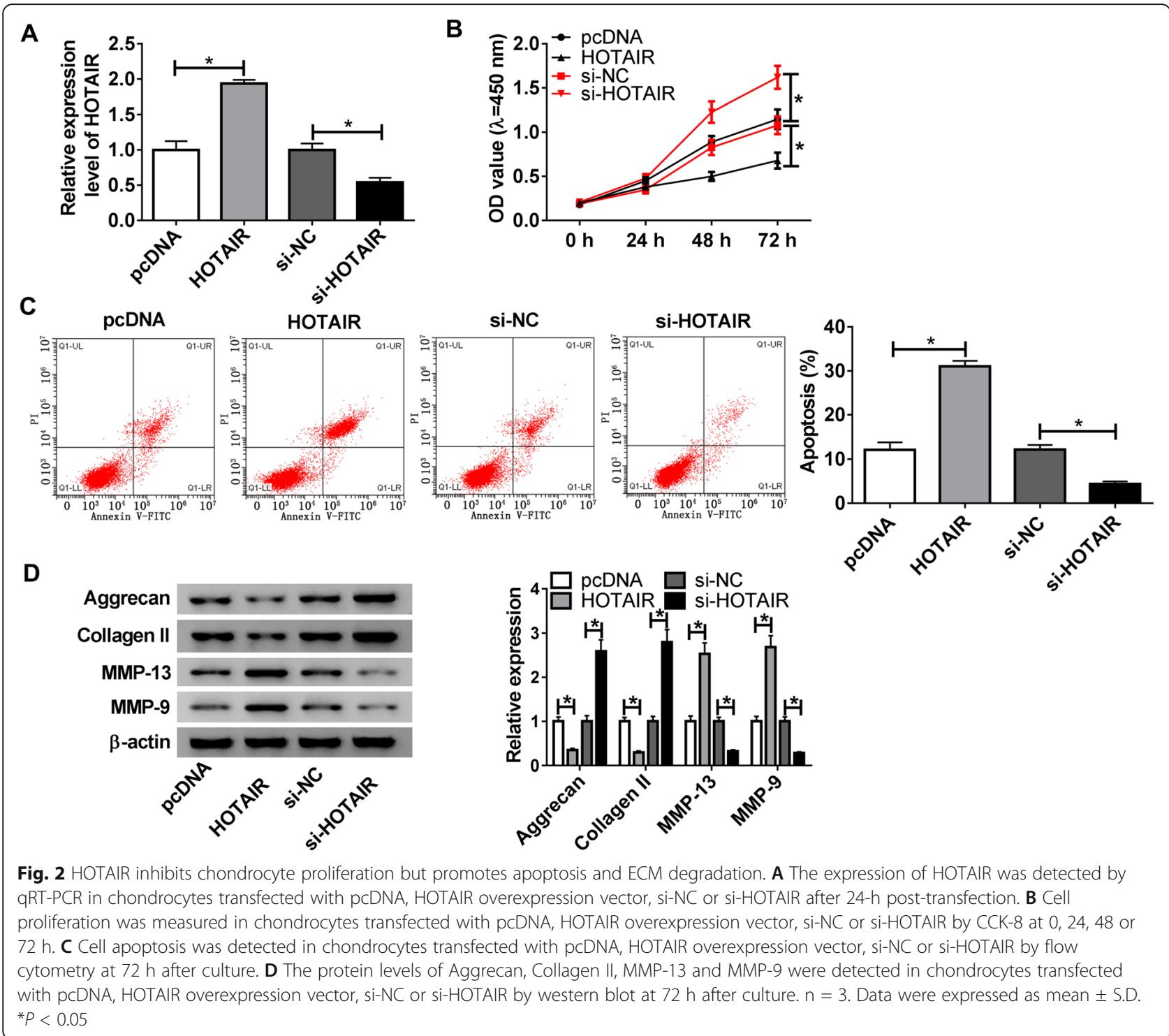

\section{HOTAIR regulates CXCL12 expression to mediate} chondrocyte progression by sponging miR-107

To further explore the mechanism in this study, bioinformatics analysis was performed using DIANA tools. Six upregulated targets (CXCL12, FUT2, FUT1, EIF4G2, EZH2 and PTEN) of miR-107 were selected according to previous reports $[14,16,20,23,27,28]$. Moreover, CXCL12 abundance was reduced most via miR-107 overexpression than other 5 mRNAs (Supplementary Figure 1B). Hence, CSCL12 was selected for further study. The potential binding sites of miR-107 and CXCL12 are shown in Fig. 5A. The luciferase reporter assay demonstrated that CXCL12 was a target of miR107 , revealed by effect of miR-107 on luciferase activity (Fig. 5B, C). Furthermore, the expression of CXCL12 was measured in OA and normal samples $(\mathrm{n}=23)$. As described in Figs. 5D and E, the mRNA and protein levels of CXCL12 were abnormally enhanced in OA cartilage compared with that in the normal group. Meanwhile, the expression of CXCL12 in OA cartilage was negatively associated with miR-107 level $(\mathrm{r}=-0.8225, P$ $<0.0001$ ) (Fig. 5F), but positively correlated with HOTAIR abundance $(\mathrm{r}=0.8154, P<0.0001)$ (Fig. 5G). In addition, the expression of CXCL12 protein was negatively regulated by miR-107 overexpression or HOTAIR interference (Fig. 5H, I).

To investigate whether CXCL12 was involved in the HOTAIR-mediated mechanism, its abundance was overexpressed in chondrocytes using the overexpression vector on the basis of miR-107 overexpression or HOTAIR silence. As shown in Figs. 6A and B, overexpression of miR-107 promoted chondrocyte proliferation and inhibited apoptosis. Moreover, miR-107 resulted in the upregulation of Aggrecan and Collagen II as well as the 


\section{A}

\section{hsa-miR-107 3' ACUAUCGGGACAUGUUACGACGA 5' HOTAIR WT 5'...UAUGCAGUGGGACCCUGCUGCA...3' \\ HOTAIR MUT}

B

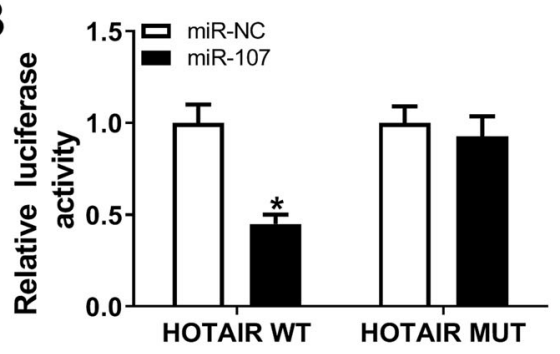

D

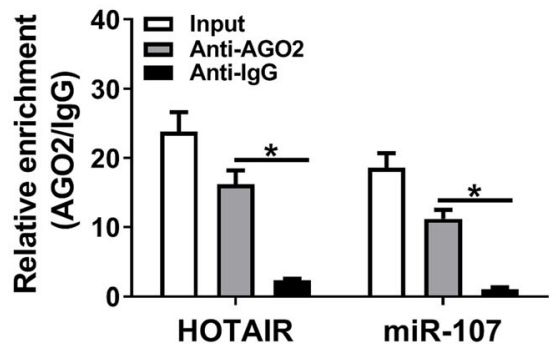

C

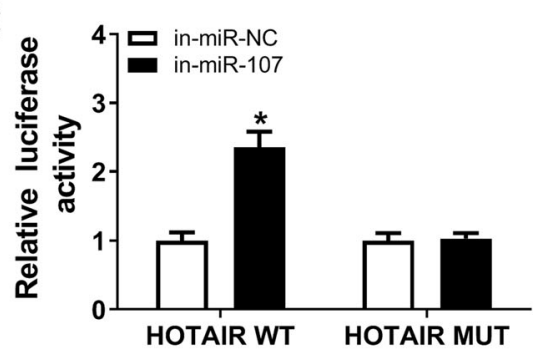

E

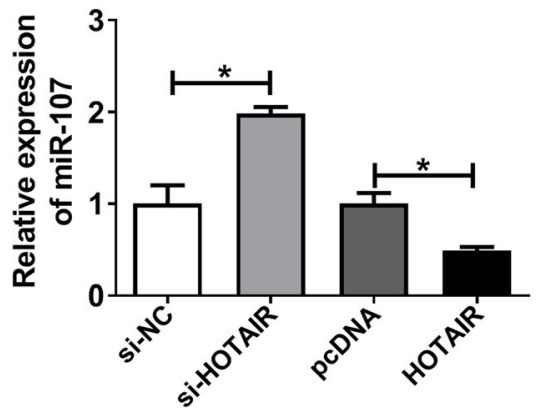

Fig. 3 HOTAIR is a decoy of miR-107. A The binding sites of HOTAIR and miR-107 were predicted via DIANA tools. B and C Luciferase activity was measured in chondrocytes co-transfected with HOTAIR WT or HOTAIR MUT and miR-NC, miR-107 mimic, in-miR-NC, or in-miR-107 at 24 h posttransfection. D The levels of HOTAIR and miR-107 were measured after enrichment of Ago2 or lgG RIP for $8 \mathrm{~h}$ by qRT-PCR. E The expression of miR-107 was detected in chondrocytes transfected with si-NC, si-HOTAIR, pCDNA or HOTAIR overexpression vector by qRT-PCR at $24 \mathrm{~h}$ posttransfection. $\mathrm{n}=3$. Data were expressed as mean \pm S.D. ${ }^{*} P<0.05$

downregulation of MMP-13 and MMP-9 in chondrocytes (Fig. 6C). Additionally, these events were weakened by restoration of CXCL12 (Fig. 6A-C). Besides, overexpression of CXCL12 evidently overturned the regulatory role of HOTAIR knockdown in proliferation, apoptosis and ECM-related protein expression in chondrocytes (Fig. 6D-F).

Moreover, the results of qRT-PCR and western blot exhibited that CXCL12 mRNA and protein levels in chondrocytes were significantly reduced by HOTAIR silence, while this event was attenuated by exhaustion of miR-107 (Fig. 7A, B). These data reflected that HOTAIR regulated chondrocyte progression by sponging miR-107 and regulating CXCL12.

\section{Discussion}

LncRNAs play important roles in chondrogenesis and OA progression via mediating inflammatory response, ECM degradation and apoptosis $[29,30]$. This work revealed that HOTAIR expression was enhanced in OA tissues, suggesting that HOTAIR might contribute to OA progression, which is also in agreement with the previous study [31]. In this work, we focused on the role and mechanism of HOTAIR in the development of OA. Here we first demonstrated that HOTAIR knockdown inhibited chondrocyte injury by regulating miR-107/ CXCL12.

A previous report suggested that HOTAIR might promote chondrocyte apoptosis and matrix metalloproteinase (MMP) expression [32]. Moreover, HOTAIR could induce cartilage degradation by activating the Wnt pathway [33]. Additionally, HOTAIR could promote chondrocyte apoptosis and ECM degradation by regulating miR-20b or miR-130a-3p in OA [34, 35]. This study described that HOTAIR suppressed chondrocyte proliferation and promoted apoptosis, which is induced by the pro-apoptotic role of HOTAIR. Moreover, the structure of ECM is required for the normal function of cartilage, and ECM degradation of chondrocytes contributes to the development of OA [36, 37]. Aggrecan and Collagen II are the constituents of ECM [38]. The degradative matrix metalloproteinases (such as MMP-13 and MMP9) are responsible for the degradation of Aggrecan and Collagen II proteins, which contribute to ECM degradation during OA pathogenesis [39, 40]. By detecting these protein expression, we found that HOTAIR 


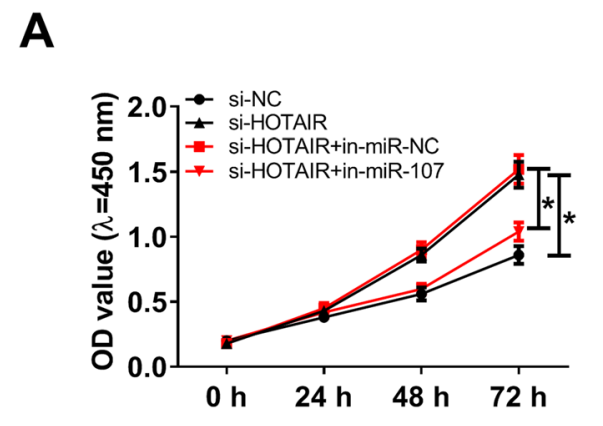

C

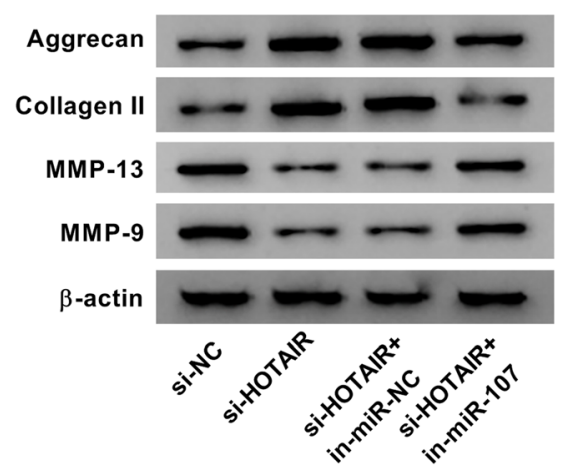

B
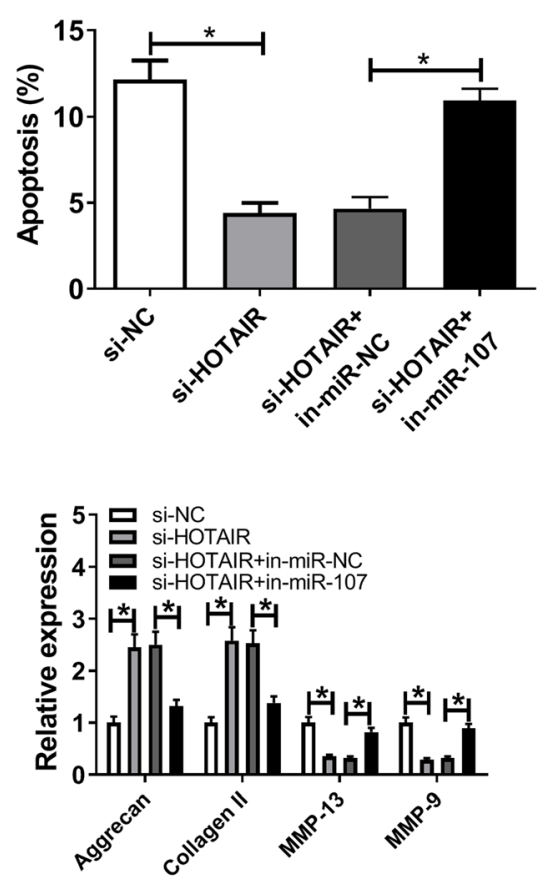

Fig. 4 Deficiency of miR-107 reverses silencing HOTAIR-mediated chondrocyte progression. A Cell proliferation was measured in chondrocytes transfected with si-NC, si-HOTAIR, si-HOTAIR + in-miR-NC or in-miR-107 by CCK-8 at 0, 24, 48 or 72 h. B Cell apoptosis was detected in chondrocytes transfected with si-NC, si-HOTAIR, si-HOTAIR + in-miR-NC or in-miR-107 by flow cytometry at $72 \mathrm{~h}$ after culture. C The protein levels of Aggrecan, Collagen II, MMP-13 and MMP-9 were examined in chondrocytes transfected with si-NC, si-HOTAIR, si-HOTAIR + in-miR-NC or inmiR-107 by western blot at $72 \mathrm{~h}$ after culture. $\mathrm{n}=3$. Data were expressed as mean \pm S.D. ${ }^{*} P<0.05$

promoted MMP-13 and MMP-9 expression but inhibited Aggrecan and Collagen II levels, indicating that HOTAIR promoted ECM degradation, which was also in agreement with the previous study [14]. Meanwhile, ECM degradation was also repressed by the knockdown of HOTAIR. These findings indicated that HOTAIR knockdown might protect against chondrocyte injury by inhibiting cell apoptosis and ECM degradation, leading to the inhibition of OA progression. However, the molecular mechanism by which HOTAIR mediates OA progression through the miRNA/mRNA axis remains unclear.

The ceRNA network is the main mechanism that allows IncRNA participating in cancer or disease progression. HOTAIR has been revealed to regulate cell proliferation, migration and invasion via functioning as a ceRNA for miRNAs, such as miR-126-5p and miR-646 $[41,42]$. Moreover, it was reported that HOTAIR could regulate miR-17-5p/FUT2 or miR-20b/PTEN axis to promote chondrocyte damage during OA [14, 34]. Our study wanted to explore an additional regulatory network mediated by this IncRNA. Here we firstly confirmed miR-107 was targeted by HOTAIR. miR-107 expression is declined in OA cartilage, and its overexpression promoted cell proliferation but suppressed apoptosis and ECM degradation, suggesting the protecting role of miR-107 in OA chondrocytes, which was consistent with a former research [17]. The negative correlation between HOTAIR and miR-107 expression levels stimulated our interest to analyze whether HOTAIR regulated miR-107 on a ceRNA-based mechanism. This study demonstrated that HOTAIR is a decoy of miR-107, which was validated by the luciferase reporter assay and RIP. In addition, knockdown of miR107 abrogated silencing HOTAIR-mediated inhibition of chondrocyte injury, indicating that HOTAIR regulated OA progression by sponging miR-107.

To further elucidate the ceRNA network, the target of miR-107 was explored. This study first confirmed that CXCL12 was targeted by miR-107 using the luciferase reporter assay. CXCL12 could mediate CXCR4 to activate inflammatory signaling to induce chondrocyte apoptosis or promote the secretion of MMPs to degrade the surrounding ECM [43-45]. Previous studies reported that CXCL12 could promote OA progression by decreasing chondrocyte proliferation and promoting ECM degradation $[27,46]$. In this study, our findings exhibited that CXCL12 mitigated the effect of miR-107 
A

hsa-miR-107

CXCL12 3'UTR WT

CXCL12 3'UTR MUT

B

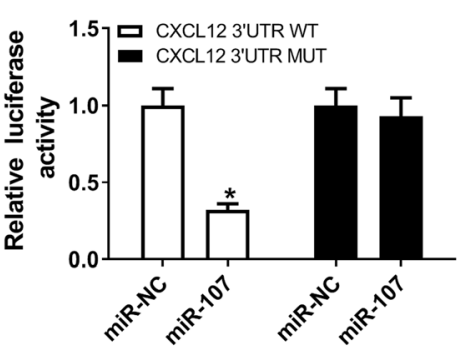

D

$\mathbf{F}$
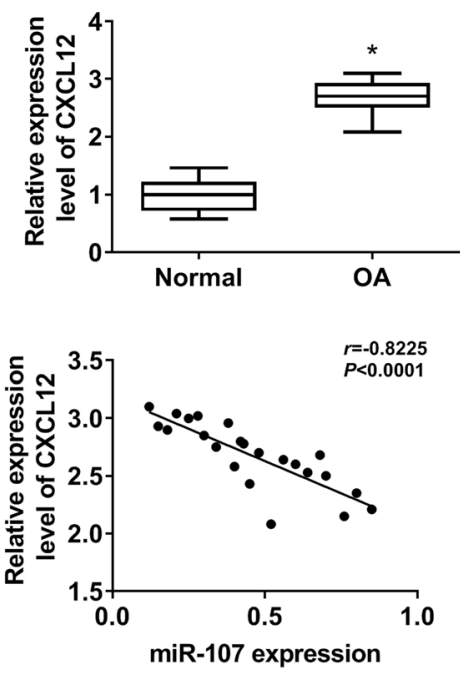

H

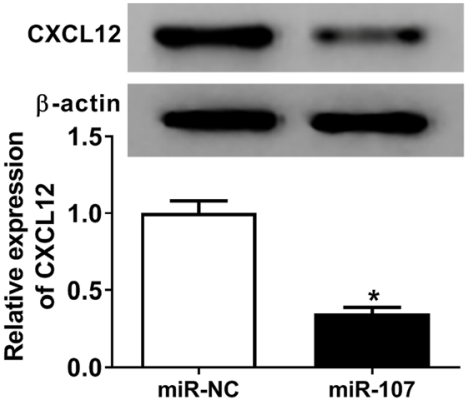

3' ACUAUCGGGACAUGUUACGACGA 5' 5'...CCCUCCGGGACCUGGUGCUGCC....3' 5 '...CCCUCCGGGACCUGGACGACGC...3'

C

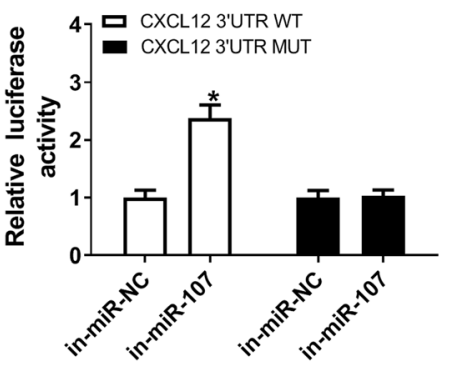

E

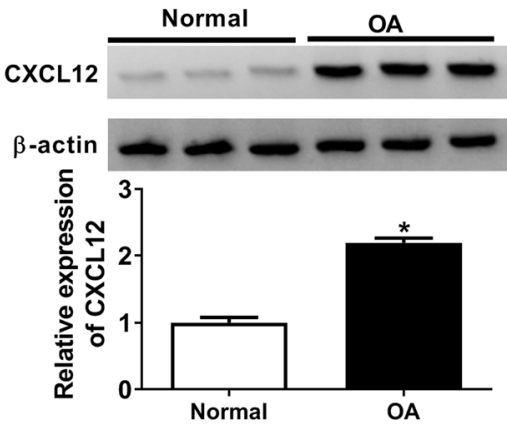

G
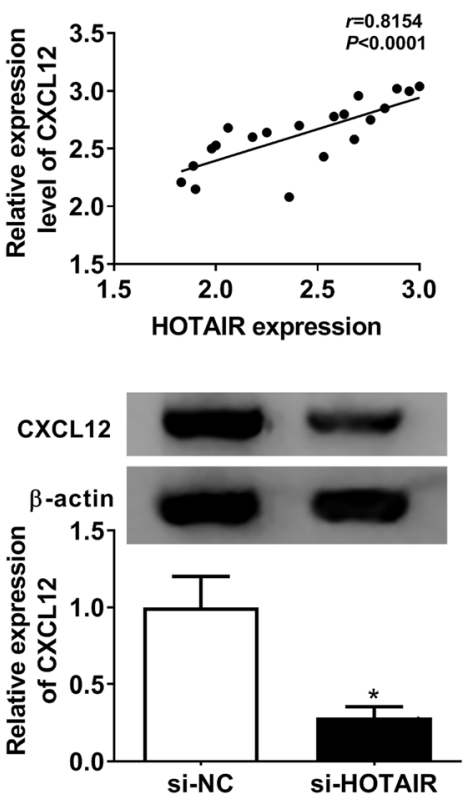

Fig. $\mathbf{5}$ CXCL12 is a target of miR-107. A The binding sites of miR-107 and CXCL12 were predicted via DIANA tools. B and C Luciferase activity was measured in chondrocytes co-transfected with CXCL12 3'UTR WT or CXCL12 3'UTR MUT and miR-NC, miR-107 mimic, in-miR-NC or in-miR-107 at $24 \mathrm{~h}$ post-transfection. $\mathbf{D}$ and $\mathbf{E}$ The expression of CXCL12 at mRNA and protein levels was measured in OA and normal samples by qRT-PCR and western blot. $\mathbf{F}$ and $\mathbf{G}$ The correlation of abundance of CXCL12 and miR-107 or HOTAIR in OA tissues was evaluated by Spearman's correlation analysis. $\mathbf{H}$ The protein level of CXCL12 was measured in chondrocytes transfected with miR-NC or miR-107 mimic by western blot at $24 \mathrm{~h}$ posttransfection. I The protein level of CXCL12 was detected in chondrocytes transfected with si-NC or si-HOTAIR by western blot at $24 \mathrm{~h}$ posttransfection. $\mathrm{n}=3$. Data were expressed as mean \pm S.D. ${ }^{*} P<0.05$ 

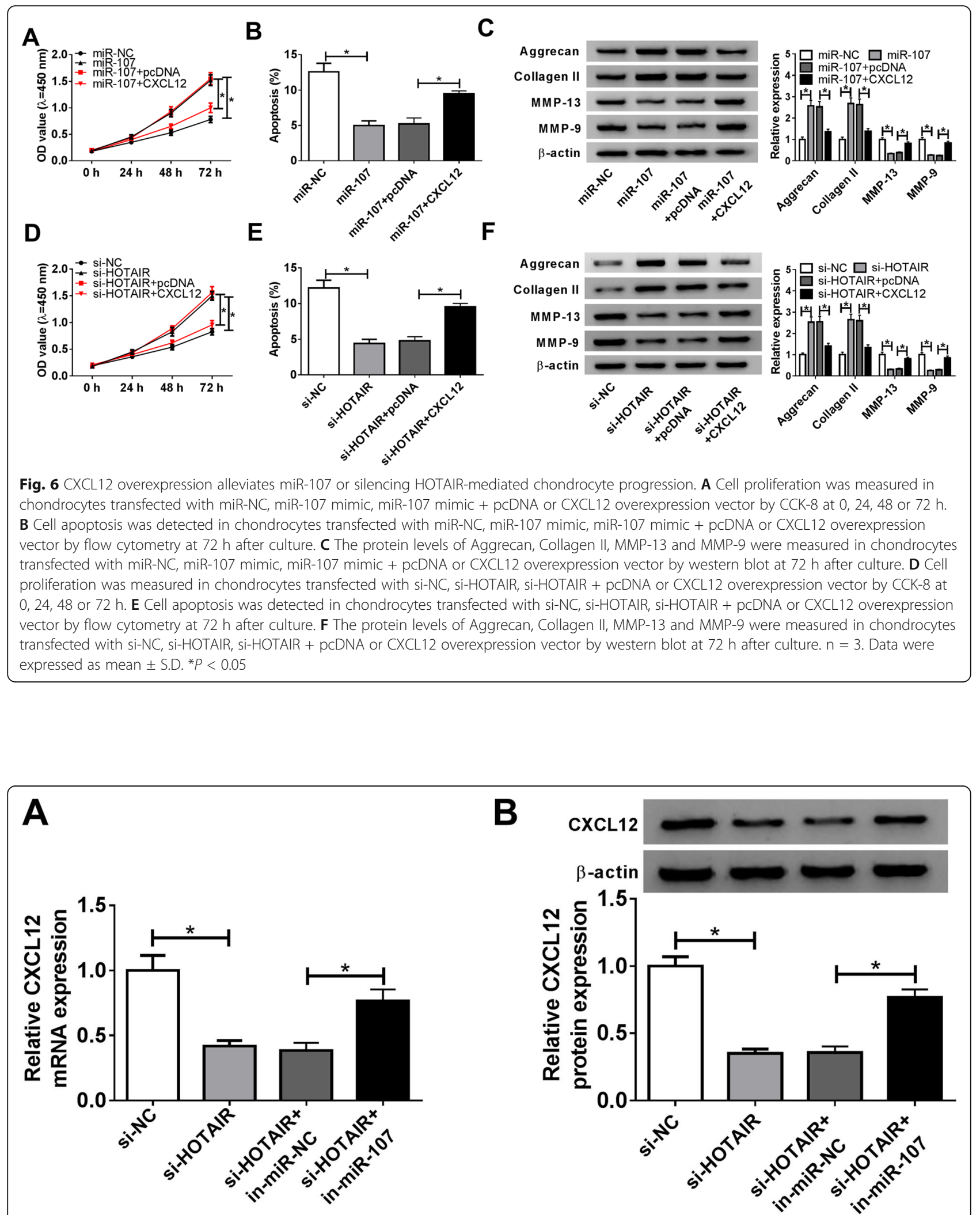

Fig. 7 HOTAIR regulates CXCL12 expression by competitively sponging miR-107. A and $\mathbf{B}$ The abundances of CXCL12 at mRNA and protein levels were measured in chondrocytes transfected with si-NC, si-HOTAIR, si-HOTAIR + in-miR-NC or in-miR-107 by qRT-PCR and western blot at $24 \mathrm{~h}$ post-transfection. $n=3$. Data were expressed as mean \pm S.D. ${ }^{*} P<0.05$ 
overexpression and HOTAIR knockdown on chondrocyte proliferation, apoptosis and ECM degradation, indicating the important role of CXCL12 in OA progression. Furthermore, we found that knockdown of HOTAIR decreased CXCL12 expression in chondrocytes. However, this effect was attenuated by downregulation of miR107, indicating that HOTAIR regulated CXCL12 expression by modulating miR-107.

However, there were some limitations in our study. Previous studies reported that the chondrocytes treated via IL-1 $\beta$, TNF- $\alpha$ or LPS were widely used to mimic the OA model $[47,48]$, and some studies have used U937activated monocyte-conditioned medium to stimulate OA chondrocytes to induce OA [49]. Our research uses untreated OA chondrocytes for research, which may not completely mimic the inflammatory environment of OA. In future studies, we will simulate the inflammatory environment of $\mathrm{OA}$ in vitro to further confirm our conclusions. Additionally, the upstream and downstream effectors of HOTAIR/miR-107/CXCL12 in OA progression were not explored in this study, which should be explored in further study. Furthermore, the in vivo experimental models were absent in the current research. Hence, an animal model of OA should be established to further investigate this network in future.

In short, HOTAIR was highly expressed in OA cartilage, and its knockdown inhibited the dysfunction of chondrocytes by promoting chondrocyte proliferation and suppressing apoptosis and ECM degradation. We further confirmed this effect might require miR-107/ CXCL12 axis. This elucidates a new mechanism underlying chondrocyte damage, which might indicate a novel insight into OA progression and provide novel target for the treatment of OA.

\section{Supplementary Information}

The online version contains supplementary material available at https://doi. org/10.1186/s13018-021-02547-7.

Additional file 1 Supplementary Figure 1 The effect of HOTAIR or miR-107 on downstream target levels. (A) MiR-107, miR-197-3p, miR136-5p, miR-211-5p and miR-17-5p levels were detected in chondrocytes transfected with pcDNA or HOTAIR overexpression vector at $24 \mathrm{~h}$ posttransfection. (B) CXCL12, FUT2, FUT1, EIF4G2, EZH2 and PTEN levels were measured in chondrocytes transfected with miR-NC or miR-107 mimic at 24 h post-transfection. $n=3$. Data were expressed as mean \pm S.D. ${ }^{*} P<0.05$.

\section{Acknowledgements}

Not applicable

\section{Authors' contributions}

Experiments were designed by JPL and YX, and were performed by ZXW. $J P L$ and ZXW analyzed data. YX interpreted results of experiments. The manuscript was written by $Y X$. The authors read and approved the final manuscript.

\section{Funding}

No funding was received.
Availability of data and materials

The analyzed data sets generated during the present study are available from the corresponding author on reasonable request.

\section{Declarations}

Ethics approval and consent to participate

The present study was approved by the ethical review committee of Yan'an Hospital Affiliated to Kunming Medical University. Written informed consent was obtained from all enrolled patients.

\section{Consent for publication}

Patients agreed to participate in this work.

\section{Competing interests}

The authors declare that they have no competing interests.

Received: 16 March 2021 Accepted: 8 June 2021

Published online: 28 June 2021

\section{References}

1. Belluzzi E, et al. Infrapatellar fat pad gene expression and protein production in patients with and without osteoarthritis. Int J Mol Sci. 2020; 21(17):6016. https://doi.org/10.3390/ijms21176016.

2. Loeser RF, Goldring SR, Scanzello CR, Goldring MB. Osteoarthritis: a disease of the joint as an organ. Arthritis Rheum. 2012;64(6):1697-707. https://doi.org/1 0.1002 /art.34453.

3. Aigner T, Söder S, Gebhard PM, McAlinden A, Haag J. Mechanisms of disease: role of chondrocytes in the pathogenesis of osteoarthritis--structure, chaos and senescence. Nat Clin Pract Rheumatol. 2007;3(7):391-9. https://doi.org/10.103 8/ncprheum0534.

4. Zamli Z, Adams M, Tarlton J, Sharif M. Increased chondrocyte apoptosis is associated with progression of osteoarthritis in spontaneous Guinea pig models of the disease. Int J Mol Sci. 2013;14(9):17729-43. https://doi.org/1 0.3390/ijms140917729.

5. Charlier E, Deroyer C, Ciregia F, Malaise O, Neuville S, Plener Z, et al. Chondrocyte dedifferentiation and osteoarthritis (OA). Biochem Pharmacol. 2019;165:49-65. https://doi.org/10.1016/j.bcp.2019.02.036.

6. Razmara E, et al. Non-coding RNAs in cartilage development: an updated review. Int J Mol Sci. 2019;20(18):4475. https://doi.org/10.3390/ijms20184475.

7. Jiang S, Liu Y, Xu B, Zhang Y, Yang M. Noncoding RNAs: new regulatory code in chondrocyte apoptosis and autophagy. Wiley Interdiscip Rev RNA. 2020; 11(4):e1584. https://doi.org/10.1002/wrna.1584

8. Zhu J, et al. IncRNAs: function and mechanism in cartilage development, degeneration, and regeneration. Stem Cell Res Ther. 2019;10(1):344.

9. $\quad$ Song W, Xie J, Li J, Bao C, Xiao Y. The emerging roles of long noncoding RNAs in bone homeostasis and their potential application in bone-related diseases. DNA Cell Biol. 2020;39(6):926-37. https://doi.org/10.1089/dna.2020. 5391.

10. Tu J, Huang W, Zhang W, Mei J, Zhu C. The emerging role of IncRNAs in chondrocytes from osteoarthritis patients. Biomed Pharmacother. 2020;131: 110642. https://doi.org/10.1016/j.biopha.2020.110642.

11. Dong $X$, He X, Guan A, Huang W, Jia H, Huang $Y$, et al. Long non-coding RNA Hotair promotes gastric cancer progression via miR-217-GPC5 axis. Life Sci. 2019;217:271-82. https://doi.org/10.1016/j.lfs.2018.12.024.

12. Chen SS, Peng M, Zhou GZ, Pu YC, Yi MC, Zhu Y, et al. Long non-coding RNA HOTAIR regulates the development of non-small cell lung cancer through miR-217/DACH1 signaling pathway. Eur Rev Med Pharmacol Sci. 2019;23(2):670-8. https://doi.org/10.26355/eurrev_201901_16905.

13. Jiang $M$, et al. LnCRNA PACER is down-regulated in osteoarthritis and regulates chondrocyte apoptosis and IncRNA HOTAIR expression. Biosci Rep. 2019;39(6):BSR20190404. https://doi.org/10.1042/BSR20190404.

14. Hu J, Wang Z, Shan Y, Pan Y, Ma J, Jia L. Long non-coding RNA HOTAIR promotes osteoarthritis progression via miR-17-5p/FUT2/beta-catenin axis. Cell Death Dis. 2018;9(7):711. https://doi.org/10.1038/s41419-018-0746-z.

15. Malemud CJ. MicroRNAs and osteoarthritis. Cells. 2018;7(8):92. https://doi. org/10.3390/cells7080092.

16. Tian F, Wang J, Zhang Z, Yang J. miR-107 modulates chondrocyte proliferation, apoptosis, and extracellular matrix synthesis by targeting PTEN. Int J Clin Exp Pathol. 2019;12(2):488-97. 
17. Zhao X, Li H, Wang L. MicroRNA-107 regulates autophagy and apoptosis of osteoarthritis chondrocytes by targeting TRAF3. Int Immunopharmacol. 2019;71:181-7. https://doi.org/10.1016/j.intimp.2019.03.005.

18. Zhou J, Li C, Yu A, Jie S, du X, Liu T, et al. Bioinformatics analysis of differentially expressed genes involved in human developmental chondrogenesis. Medicine (Baltimore). 2019;98(27):e16240. https://doi.org/1 0.1097/MD.0000000000016240.

19. Werner L, Guzner-Gur H, Dotan I. Involvement of CXCR4/CXCR7/CXCL12 interactions in inflammatory bowel disease. Theranostics. 2013;3(1):40-6. https://doi.org/10.7150/thno.5135.

20. Wang Z, Hu J, Pan Y, Shan Y, Jiang L, Qi X, et al. miR-140-5p/miR-149 affects chondrocyte proliferation, apoptosis, and autophagy by targeting FUT1 in osteoarthritis. Inflammation. 2018;41(3):959-71. https://doi.org/10.1007/s1 0753-018-0750-6.

21. Liu X, Gao F, Wang W, Yan J. Expression of miR-204 in patients with osteoarthritis and its damage to chondrocytes. J Musculoskelet Neuronal Interact. 2020;20(2):265-71.

22. Livak KJ, Schmittgen TD. Analysis of relative gene expression data using real-time quantitative PCR and the 2(-Delta Delta C(T)) method. Methods. 2001;25(4):402-8. https://doi.org/10.1006/meth.2001.1262.

23. Gao S, et al. MicroRNA-197 regulates chondrocyte proliferation, migration, and inflammation in pathogenesis of osteoarthritis by targeting EIF4G2. Biosci Rep. 2020:40(9):BSR20192095. https://doi.org/10.1042/BSR20192095.

24. Chen X, Shi Y, Xue P, Ma X, Li J, Zhang J. Mesenchymal stem cell-derived exosomal microRNA-136-5p inhibits chondrocyte degeneration in traumatic osteoarthritis by targeting ELF3. Arthritis Res Ther. 2020;22(1):256. https://doi. org/10.1186/s13075-020-02325-6.

25. Liu H, Luo J. miR-211-5p contributes to chondrocyte differentiation by suppressing Fibulin-4 expression to play a role in osteoarthritis. J Biochem. 2019;166(6):495-502. https://doi.org/10.1093/jb/mvz065.

26. Li Z, Wang J, Yang J. TUG1 knockdown promoted viability and inhibited apoptosis and cartilage ECM degradation in chondrocytes via the miR-175p/FUT1 pathway in osteoarthritis. Exp Ther Med. 2020;20(6):154. https://doi. org/10.3892/etm.2020.9283

27. Dai Y, Liu S, Xie X, Ding M, Zhou Q, Zhou X. MicroRNA31 promotes chondrocyte proliferation by targeting CXC motif chemokine ligand 12. Mol Med Rep. 2019;19(3):2231-7. https://doi.org/10.3892/mmr.2019.9859.

28. Chen $L$, Wu Y, Wu Y, Wang Y, Sun L, Li F. The inhibition of EZH2 ameliorates osteoarthritis development through the Wnt/beta-catenin pathway. Sci Rep. 2016;6(1):29176. https://doi.org/10.1038/srep29176.

29. Sun H, Peng G, Ning X, Wang J, Yang H, Deng J. Emerging roles of long noncoding RNA in chondrogenesis, osteogenesis, and osteoarthritis. Am J Transl Res. 2019;11(1):16-30.

30. Chen WK, et al. IncRNAs: novel players in intervertebral disc degeneration and osteoarthritis. Cell Prolif. 2017;50(1):e12313. https://doi.org/10.1111/ cpr.12313.

31. Xing D, Liang JQ, Li Y, Lu J, Jia HB, Xu LY, et al. Identification of long noncoding RNA associated with osteoarthritis in humans. Orthop Surg. 2014;6(4):288-93. https://doi.org/10.1111/os.12147.

32. Zhang $C$, Wang $P$, Jiang $P, L v Y$, Dong $C$, Dai $X$, et al. Upregulation of IncRNA HOTAIR contributes to IL-1 beta-induced MMP overexpression and chondrocytes apoptosis in temporomandibular joint osteoarthritis. Gene. 2016;586(2):248-53. https://doi.org/10.1016/.j.gene.2016.04.016.

33. Yang $Y$, Xing D, Wang Y, Jia H, Li B, Li JJ. A long non-coding RNA, HOTAIR, promotes cartilage degradation in osteoarthritis by inhibiting WIF-1 expression and activating Wnt pathway. BMC Mol Cell Biol. 2020;21(1):53. https://doi.org/10.1186/s12860-020-00299-6.

34. Chen Y, Zhang L, Li E, Zhang G, Hou Y, Yuan W, et al. Long-chain noncoding RNA HOTAIR promotes the progression of osteoarthritis via sponging miR-20b/PTEN axis. Life Sci. 2020;253:117685. https://doi.org/10.1 016/j.lfs.2020.117685.

35. He B, Jiang D. HOTAIR-induced apoptosis is mediated by sponging miR$130 a-3 p$ to repress chondrocyte autophagy in knee osteoarthritis. Cell Biol Int. 2020;44(2):524-35. https://doi.org/10.1002/cbin.11253.

36. Krishnan Y, Grodzinsky AJ. Cartilage diseases. Matrix Biol. 2018;71-72:51-69. https://doi.org/10.1016/j.matbio.2018.05.005.

37. Liu C, Wang B, Xiao L, Li Y, Xu L, Zhao Z, et al. Protective effects of the pericellular matrix of chondrocyte on articular cartilage against the development of osteoarthritis. Histol Histopathol. 2018;33(8):757-64. https:// doi.org/10.14670/HH-11-967.
38. Shahid M, Manchi G, Slunsky P, Naseer O, Fatima A, Leo B, et al. A systemic review of existing serological possibilities to diagnose canine osteoarthritis with a particular focus on extracellular matrix proteoglycans and protein. Pol J Vet Sci. 2017:20(1):189-201. https://doi.org/10.1515/pjvs-2017-0024.

39. Malemud CJ, et al. Matrix metalloproteinase- 9 production by immortalized human chondrocyte lines. J Clin Cell Immunol. 2016;7(3):422. https://doi. org/10.4172/2155-9899.1000422.

40. Mehana EE, Khafaga AF, El-Blehi SS. The role of matrix metalloproteinases in osteoarthritis pathogenesis: an updated review. Life Sci. 2019;234:116786. https://doi.org/10.1016/j.lfs.2019.116786

41. Lin $\mathrm{Q}$, et al. LncRNA HOTAIR targets miR-126-5p to promote the progression of Parkinson's disease through RAB3IP. Biol Chem. 2018;400(9):1217-28. https://doi.org/10.1515/hsz-2018-0431.

42. Zhou YX, Wang C, Mao LW, Wang YL, Xia LQ, Zhao W, et al. Long noncoding RNA HOTAIR mediates the estrogen-induced metastasis of endometrial cancer cells via the miR-646/NPM1 axis. Am J Physiol Cell Physiol. 2018;314(6):C690-701. https://doi.org/10.1152/ajpcell.00222.2017.

43. Zheng X, Zhao FC, Pang Y, Li DY, Yao SC, Sun SS, et al. Downregulation of miR-221-3p contributes to IL-1 beta-induced cartilage degradation by directly targeting the SDF1/CXCR4 signaling pathway. J Mol Med (Berl). 2017;95(6):615-27. https://doi.org/10.1007/s00109-017-1516-6.

44. Jia D, Li Y, Han R, Wang K, Cai G, He C, et al. miR146a5p expression is upregulated by the CXCR4 antagonist TN14003 and attenuates SDF1induced cartilage degradation. Mol Med Rep. 2019:19(5):4388-400. https://doi.org/10.3892/mmr.2019.10076.

45. Oin HJ, Xu T, Wu HT, Yao ZL, Hou YL, Xie YH, et al. SDF-1/CXCR4 axis coordinates crosstalk between subchondral bone and articular cartilage in osteoarthritis pathogenesis. Bone. 2019;125:140-50. https://doi.org/10.1016/j. bone.2019.05.010

46. Lu W, et al. CXCL12/CXCR4 axis regulates aggrecanase activation and cartilage degradation in a post-traumatic osteoarthritis rat model. Int J Mol Sci. 2016;17(10):1522. https://doi.org/10.3390/ijms17101522.

47. Yu D, et al. Upregulated ox 40 l can be inhibited by miR-146a-5p in condylar chondrocytes induced by IL-1 beta and TNF-alpha: a possible regulatory mechanism in osteoarthritis. Int Arch Allergy Immunol. 2020;182(5):408-16. https://doi.org/10.1159/000512291.

48. Zhang G, Zhang Q, Zhu J, Tang J, Nie M. LncRNA ARFRP1 knockdown inhibits LPS-induced the injury of chondrocytes by regulation of NF-kappaB pathway through modulating miR-15a-5p/TLR4 axis. Life Sci. 2020;261: 118429. https://doi.org/10.1016/.lfs.2020.118429.

49. Tarricone $\mathrm{E}$, et al. Anti-inflammatory performance of lactose-modified chitosan and hyaluronic acid mixtures in an in vitro macrophage-mediated inflammation osteoarthritis model. Cells. 2020;9(6):1328. https://doi.org/10.33 90/cells9061328.

\section{Publisher's Note}

Springer Nature remains neutral with regard to jurisdictional claims in published maps and institutional affiliations.

Ready to submit your research? Choose BMC and benefit from:

- fast, convenient online submission

- thorough peer review by experienced researchers in your field

- rapid publication on acceptance

- support for research data, including large and complex data types

- gold Open Access which fosters wider collaboration and increased citations

- maximum visibility for your research: over $100 \mathrm{M}$ website views per year

At BMC, research is always in progress.

Learn more biomedcentral.com/submissions 